\title{
BROADBAND TRAILING EDGE NOISE PREDICTIONS IN THE TIME DOMAIN
}

\author{
J. Casper* and F. Farassat ${ }^{\dagger}$ \\ NASA Langley Research Center \\ Hampton, VA 23681
}

\author{
REVISED \\ for Publication in \\ Journal of Sound and Vibration
}

February 15, 2003

Suggested Running Head: TRAILING EDGE NOISE

Pages: 38

Tables: 0

Figures: 13
Send proofs to: Dr. Jay H. Casper
Mail Stop 128
NASA Langley Research Center
Hampton, VA 23681

\footnotetext{
* Research Scientist, Computational Modeling and Simulation Branch, Mail Stop 128, NASA Langley Research Center, Hampton, VA 23681, j.h.casper@larc.nasa.gov

$\dagger$ Senior Research Scientist, Aeroacoustics Branch, Mail Stop 461, NASA Langley Research Center, Hampton, VA 23681, f.farassat@larc.nasa.gov
} 


\begin{abstract}
A recently developed analytic result in acoustics, "Formulation 1B," is used to compute broadband trailing edge noise from an unsteady surface pressure distribution on a thin airfoil in the time domain. This formulation is a new solution of the Ffowcs Williams-Hawkings equation with the loading source term, and has been shown in previous research to provide time domain predictions of broadband noise that are in excellent agreement with experimental results. Furthermore, this formulation lends itself readily to rotating reference frames and statistical analysis of broadband trailing edge noise. Formulation 1B is used to calculate the far field noise radiated from the trailing edge of a NACA 0012 airfoil in low Mach number flows, by using both analytical and experimental data on the airfoil surface. The acoustic predictions are compared with analytical results and experimental measurements that are available in the literature. Good agreement between predictions and measurements is obtained.
\end{abstract}




\section{INTRODUCTION}

Trailing edge (TE) noise has been the subject of extensive research within the aeroacoustic community for decades, both experimentally and analytically. Areas of current research include the prediction of TE noise from rotating machinery and airframes. Research in the area of TE noise prediction has, in large part, been motivated by the desire to incorporate the results of TE noise analysis into a design methodology. The present work is similarly motivated, and the resulting formulation should lend itself well to an engineering design tool suite when aeroacoustics plays a role in the design.

The literature abounds with various theoretical approaches to TE noise prediction. Howe [1] categorized the various theories of TE noise into three groups:

i. Theories based on the Lighthill [2] acoustic analogy, e.g., Ffowcs Williams and Hall [3].

ii. Theories based on the solution of special problems approximated by the linearized hydrodynamics equations, e.g., Amiet $[4,5]$ and Goldstein [6].

iii. Ad hoc models involving postulated source distributions for which strengths and types are empirically determined.

The present work falls into the first category. "Formulation 1B," originally proposed in [7], is a solution of the loading source term of the Ffowcs Williams-Hawkings equation [8]. Such time domain methods allow a total decoupling of the acoustic signal from the aerodynamics. As such, these methods readily avail themselves to acoustic predictions that are based on input from experimental measurements or computational fluid dynamics (CFD) solutions. For example, Singer et al. [9], used a solution of the Ffowcs Williams-Hawkings equation to predict TE noise from sources that were modeled with CFD simulations. The acoustic formulation in their work is known as "Formulation 1A" [10]. What distinguishes Formulation 1B from this prior formulation is its relative simplicity, which makes it highly suitable for rotational reference frames and statistical analysis of broadband TE noise. 
In [7], Formulation 1B was applied to the prediction of far field noise due to incident turbulence on a NACA 0012 airfoil at tunnel speeds ranging from $40 \mathrm{~m} / \mathrm{s}$ to $165 \mathrm{~m} / \mathrm{s}$, and the predictions were compared with the experimental results of Paterson and Amiet [11]. The time-dependent surface pressure required as input to Formulation 1B was generated by stochastic modeling of the incident turbulence and approximation of the airfoil response with a result from thin airfoil theory [12]. Formulation $1 \mathrm{~B}$ was then used to predict the acoustic pressure as a function in time at a prescribed microphone location. The time domain results were then Fourier analyzed to determine the narrowband spectra of the far field noise. The far field spectra were found to be in excellent agreement with the frequency domain predictions and experimental measurements of Paterson and Amiet [11].

In the present work, the time domain approach described in [7] is used to predict far field radiation from the trailing edge of an airfoil. In the following section, Formulation $1 \mathrm{~B}$ is briefly reviewed for the case of a flat surface in a general nonuniform motion. Some advantages of this new formulation relative to other solutions of the Ffowcs Williams-Hawkings equation are described.

In Section 3, a model problem is considered in which a single-frequency surface pressure induces an acoustic source at the trailing edge of a flat plate in uniform motion. The unsteady surface pressure is an analytical result from thin airfoil theory that is taken from the work of Amiet [4, $5,12]$. Two simple test cases are presented for validation purposes. The directivity of the tone induced by this surface pressure is examined for qualitative correctness. The results of a velocity scaling exercise are shown to be consistent with the results of Ffowcs Williams and Hall [3].

In Section 4, the surface pressure formulation introduced in Section 3 is extended by linear superposition to provide an analytic source model for broadband TE noise. This stochastically modeled surface pressure is used as input to Formulation $1 \mathrm{~B}$ to predict broadband TE noise from a NACA 0012 airfoil in a low-turbulence uniform mean flow. The surface pressure correlations that are required in the aerodynamic model are taken from two sources: an empirical flat plate formulation $[13,14]$ and experimental data [15]. The resulting calculations are compared with the acoustic predictions of Schlinker and Amiet [16] and the experimental measurements of Brooks and 
Hodgson [17].

\section{ACOUSTIC FORMULATION}

In [7], Formulation 1B was derived for the case of a flat plate in a general planar motion. Only the result is presented here; in order to do so, the following discussion is required.

Consider a flat, finite surface moving in the plane $x_{3}=0$ along a velocity vector $\vec{V}$. The velocity vector $\vec{V}$ and the surface geometry are related to the coordinate axes as pictured in Fig. 1. Let $\tilde{f}\left(x_{1}, x_{2}, t\right)$ denote a geometric function where $\tilde{f}=0$ on the surface edge and $\tilde{f}>0$ on the interior of the surface. Let $\hat{\nu}=\vec{\nabla} \tilde{f}$ denote the unit normal that lies in the plane of the surface, is normal to the edge, and is directed inward (Fig. 1). Note that $\vec{V}$ need not be constant in space or time. The only stipulation on the velocity is that the motion of the surface is in the same plane as the surface.

Let $\vec{x}=\left[x_{1}, x_{2}, x_{3}\right]^{T}$ denote the position of an observer, and $\vec{y}=\left[y_{1}, y_{2}, 0\right]^{T}$ denote the position of a source point on the surface (Fig. 1). The spatial frame of reference is fixed to the undisturbed medium. The unsteady surface pressure $p(\vec{y}, \tau)$ gives rise to sound that initiates at source time $\tau$ and radiates to the observer along $\vec{r}=\vec{x}-\vec{y}$. This sound is denoted by $p^{\prime}(\vec{x}, t)$, the perturbation pressure that arrives at the point $\left(x_{1}, x_{2}, x_{3}\right)$ at time $t$. Formulation $1 \mathrm{~B}$ predicts this sound by:

$$
\begin{aligned}
4 \pi p^{\prime}(\vec{x}, t)= & \int_{\tilde{f}>0}\left[\frac{(\partial p / \partial \tau-V \partial p / \partial s) \cos \theta}{c_{0} r\left(1-M_{r}\right)}\right]_{\mathrm{ret}} d S \\
& +\int_{\tilde{f}>0}\left[\frac{p \cos \theta}{r^{2}\left(1-M_{r}\right)}\right]_{\mathrm{ret}} d S-\int_{\tilde{f}=0}\left[\frac{M_{\nu} p \cos \theta}{r\left(1-M_{r}\right)}\right]_{\mathrm{ret}} d \ell,
\end{aligned}
$$

where $M_{\nu}$ and $M_{r}$ are the Mach numbers in the directions of $\hat{\nu}$ and $r$, respectively. The quantity $\partial p / \partial s$ is the pressure gradient in the direction of $\vec{V}$, and $V$ is the local magnitude of $\vec{V}$. The subscript "ret" denotes evaluation at retarded time $t-r / c_{0}$.

The first and third integrals in Eq. (1) are far field terms, and the second integral is the near field term. As previously discussed in [7], the relative contributions of the terms in Eq. (1) are 
considered, under the conditions of low Mach number and an observer in the acoustic far field, i.e.,

$$
M \ll 1, \quad r \gg \lambda
$$

where $\lambda$ is a typical acoustic wavelength of interest. With respect to $M$ and $r$, the surface far field integral, i.e., the first integral in Eq. (1), is proportional to $1 / r$, whereas the second and third integrals are proportional to $1 / r^{2}$ and $M / r$, respectively. Therefore, the far field surface integral dominates the signal under the conditions in Eq. (2).

Perhaps most significant in regard to the form of Eq. (1) is that it is valid, as is, for rotating surfaces. Its predecessor, Formulation 1A [10], is significantly more complicated in its rotational form and cannot be approximated by only one surface integral in the far field. Such a significant simplification for far field calculations makes Formulation 1B more suitable for statistical analysis of broadband sources for rotating surfaces. A statistical formulation based on Eq. (1) was derived in [7]. The focus of the current work is the application of Formulation $1 \mathrm{~B}$ to TE noise in the form of Eq. (1), as will be demonstrated in the following two sections.

\section{MODEL PROBLEM - TRAILING EDGE TONE}

Any noise prediction made with Eq. (1) will be only as good as the input surface pressure $p(\vec{y}, \tau)$. The current thinking is that such time-dependent pressure data would result from experimental measurement or a CFD calculation. However, in this section, a simplified analytic expression is used for $p(\vec{y}, t)$ to serve as a model problem. A result from thin airfoil theory [4] will be used

to describe the unsteady surface pressure that is produced by the passage of a single frequency disturbance past the trailing edge of a slender airfoil. This simple surface pressure formulation will be extended to a broadband source model in the following section.

\subsection{SURFACE PRESSURE FROM THIN AIRFOIL THEORY}

The airfoil for this model problem is a rectangular flat plate in the plane $x_{3}=0$, undergoing 
a uniform rectilinear motion, as in Fig. 2. The velocity vector $\vec{V}=[-U, 0,0]^{T}$, where $U$ is a constant subsonic speed. The plate's surface and its boundary, $\tilde{f} \geq 0$, are defined by the rectangle $\left\{-C \leq x_{1} \leq 0\right\} \times\left\{-b \leq x_{2} \leq b\right\}$, with the trailing edge at $x_{1}=0$. An unsteady pressure distribution is assumed on this surface, and is analytically prescribed from thin airfoil theory, as discussed below.

Amiet [4] has proposed a formulation to model the response of an airfoil to the passage of a pressure disturbance over its trailing edge. This formulation, formally derived in [12], is based on the theory of a thin airfoil of infinite span and models the moving disturbance as stationary in the variable $x_{1}-U_{c} t$, where $U_{c}$ is the convection speed of the disturbance. The induced pressure jump on the airfoil surface can be written

$$
\Delta P\left(x_{1}, t\right)=2 P_{0} g\left(x_{1}, k_{c}\right) e^{-i k_{c}\left(x_{1}-U_{c} t\right)}
$$

where $k_{c}=\omega / U_{c}$ is the streamwise convective wave number, and $P_{0}$ is the amplitude of the disturbance. The factor of two in Eq. (3a) indicates that the pressure is assumed to be antisymmetric between the upper and lower surfaces, and this expression thereby accounts for the pressure on both sides of the airfoil, i.e., the pressure jump. Note that Eq. (3a) differs from the general form for the pressure jump in [7] because the explicit term $e^{-i k_{c} x_{1}}$ in Eq. (3a) was incorporated into the transfer function $g$ in [7]. The formulation in Eq. (3a) is used here for consistency with the TE noise research of Schlinker and Amiet [16].

The transfer function $g\left(x_{1}, k_{c}\right)$ is

$$
g\left(x_{1}, k_{c}\right)=-1+(1+i) E^{*}\left[-x_{1}\left(k_{c}+\mu(1+M)\right)\right]
$$

where $\mu=M \omega / \beta^{2} U, \beta=\sqrt{1-M^{2}}$, and the function $E^{*}$ is given by

$$
E^{*}(\xi)=\int_{0}^{\xi} \frac{e^{-i u}}{(2 \pi u)^{\frac{1}{2}}} d u \equiv \mathcal{C}(\xi)-i \mathcal{S}(\xi)
$$

The quantities $\mathcal{C}(\xi)$ and $\mathcal{S}(\xi)$ are the Fresnel cosine and sine integrals, and will be evaluated 
numerically by the formulas in [20]. The final representation for the unsteady surface pressure $p\left(y_{1}, \tau\right)$, assumed to be a real quantity, is

$$
p\left(y_{1}, \tau\right)=\Re\left\{-\Delta P\left(y_{1}, \tau\right)\right\}
$$

The pressure jump is negative in Eq. (3d) because of the orientation of upper and lower surface normals in the derivation of the acoustic formulation [7].

Note that the transfer function in Eq. (3b) represents the effect of the induced surface pressure only, and neglects the effect of the incident pressure. The neglect of the incident pressure field effect is not of concern here, as this model problem is presented for illustrative purposes only. After the initial derivation of this induced pressure formulation $[4,12]$, Amiet later altered the formulation to include the effect of the incident pressure field [5]. The effects of both induced and incident surface pressure will be employed in the broadband formulation in Section 4.

\subsection{DIRECTIVITY CALCULATION}

Using Eqs. (3a)-(3d) as the input surface pressure in Eq. (1), the directivity of a single frequency source is now examined. The flat plate has a chord length $C=0.5 \mathrm{~m}$, and a $\operatorname{span} 2 b=2.0 \mathrm{~m}$. The flow speed $U$ is determined by a freestream Mach number $M=0.2$, with $c_{0}=343 \mathrm{~m} / \mathrm{s}$. The disturbance amplitude $P_{0}$ is taken as one percent of the dynamic head $q_{0}=\rho_{0} U^{2} / 2$, with $\rho_{0}=1.23$ $\mathrm{kg} / \mathrm{m}^{3}$, and the convection speed is taken to be $U_{c}=0.8 U$. The initial surface pressure $p\left(y_{1}, 0\right)$,

$-C \leq y_{1} \leq 0$, is shown in Fig. 3. This pressure profile represents the surface pressure over the entire span at observer time $t=0$. Note, again, that the formulation in Eqs. (3a)-(3d) represents the induced surface pressure only.

The radiated noise $p^{\prime}(\vec{x}, t)$ is calculated at 360 equally spaced locations on a circular arc in the plane $x_{2}=0$. The radius of this arc emanates from the mid-span location on the trailing edge, as shown in Fig. 4. The arc trajectory $(r, \psi)$ is determined by $r=2 \mathrm{~m}$ and $0 \leq \psi \leq 2 \pi$. The surface discretization is a uniform grid of $100 \times 400$ surface elements. The directivity is determined by the peak pressure amplitude calculated at each position, during one period $T=1 / f$ for a frequency of 
$2.5 \mathrm{kHz}$, with 128 timesteps in a period. Fig. 5 shows the results, in polar form, where the notation $\left\|p^{\prime}\right\|$ is interpreted as

$$
\left\|p^{\prime}\right\|=\max _{0<t<T}\left|p^{\prime}(\vec{x}, t)\right|
$$

The upstream directivity of the major lobes is consistent with the research of previous authors, e.g., Singer, et al [8].

\subsection{VELOCITY SCALING LAW}

Attention is now turned to the way in which the intensity of the far field noise, as predicted by Eq. (1), will scale as a function of velocity, when the surface pressure is described by Eqs. (3a)-(3d). A scaling law will be determined under the assumption that the acoustic source is noncompact, i.e., $\lambda \ll C$. Furthermore, the observer is assumed to be in the acoustic and geometric far field, i.e., $r \gg \lambda$ and $r \gg C$, respectively.

Because scaling laws are typically determined for low Mach number flows $[3,19]$, the Mach number range of interest is $0.01 \leq M \leq 0.2$. The surface pressure amplitude $P_{0}$ is one percent of the dynamic head. The physical dimensions of the plate are the same as in the above directivity problem. The observer is chosen at a distance of $10 \mathrm{~m}$, directly above the trailing edge, i.e., $\vec{x}=[0,0,10]^{T}$ in meters. The calculations are performed on a $100 \times 400$ uniform surface grid.

The surface pressure in Eqs. (3a)-(3d), with a frequency of $2.5 \mathrm{kHz}$, is used as input to Eq. (1) to predict the far field sound $p^{\prime}(\vec{x}, t)$ to the observer. A separate calculation is run for each of 50 equally spaced Mach numbers between 0.01 and 0.2 . Each calculation is performed for one period with 128 timesteps. The average intensity $I(\vec{x})$ of the acoustic signal at the observer $\vec{x}$, assuming spherical spreading, is then calculated by

$$
I(\vec{x})=\frac{1}{T} \int_{0}^{T} \frac{\left[p^{\prime}(\vec{x}, t)\right]^{2} d t}{\rho_{0} c_{0}}
$$

The average acoustic intensities for this test case, as a function of Mach number, are represented as circles in Fig. 6. The slope of these results on a log-log plot can be visually determined by observing their proximity to the dotted line with a slope of exactly five. This $U^{5}$ proportionality is 
consistent with the result of Ffowcs Williams and Hall [3], as expected from the idealized conditions placed upon the calculations.

\section{BROADBAND TRAILING EDGE NOISE}

The analytic surface pressure in the previous section is extended to model a broadband trailing edge source on a slender airfoil at zero angle of attack. Following the approach of Schlinker and Amiet [16], the surface pressure correlations required as input are evaluated by flat plate theory and by experimental measurements. This broadband surface pressure is used as input to Formulation 1B to predict far field radiation in the time domain. The results are Fourier analyzed and compared with experimental TE noise spectra [17].

\subsection{EXPERIMENT DESCRIPTION}

The experiment modeled in this section is reported by Brooks and Hodgson [17]. A NACA 0012 airfoil is placed between two plates at zero angle of attack in the test section of an open jet wind tunnel. A schematic of this experimental setup is shown in Fig. 7. Noise propagates from the test section into an anechoic chamber that is instrumented with microphones.

The airfoil has a chord length of $0.6096 \mathrm{~m}$ and a span of $0.46 \mathrm{~m}$. The tunnel speeds of interest here are $38.6 \mathrm{~m} / \mathrm{s}$ and $69.5 \mathrm{~m} / \mathrm{s}$. The chord-based Reynolds numbers are 1.57 million and 2.82 million, respectively. Boundary layer tripping was applied at 15 percent chord downstream of the leading edge to ensure a spanwise uniform transition location and a fully developed turbulent boundary layer at the trailing edge.

For radiated noise measurements, eight microphones are located in the plane perpendicular to the airfoil midspan. The presence of extraneous noise sources precludes direct measurement of TE noise by a single microphone. Therefore, to evaluate the TE noise, a cross-spectral analysis of pairs of microphones was employed in a manner consistent with the coherent output power method [20, 21]. The microphone pictured in Fig. 7 represents the location for which the current predictions are made, at a distance of $1.22 \mathrm{~m}$ directly above the airfoil trailing edge. Note that a shear layer 
forms downstream of the nozzle lip, between the airfoil and the microphone. Although both the directivity and the amplitude of the TE noise are affected by refraction through this shear layer, the corrections for the microphone at this location are small enough to ignore (see [17]).

\subsection{BROADBAND ANALYSIS}

For prediction purposes, the airfoil is modeled as a flat plate in order to evaluate the unsteady surface pressure with a broadband extension of the analytic formulation in Section 3. The airfoil geometry is oriented with respect to the coordinate axes as in Fig. 2, with $\left\{-C \leq x_{1} \leq 0\right\} \times\{-b \leq$ $\left.x_{2} \leq b\right\}$, where $C=0.6096 \mathrm{~m}$ and $2 b=0.46 \mathrm{~m}$.

The surface pressure arises from boundary layer turbulence that is assumed to convect in a frozen pattern along the airfoil surface toward the trailing edge. Unlike the single frequency source in Section 3, the broadband nature of the surface pressure in the present case requires consideration of both chordwise and spanwise wave numbers, $k_{1}$ and $k_{2}$, respectively. Each Fourier component of this broadband surface pressure jump is associated with a wave number pair $\left(k_{1}, k_{2}\right)$ and can be written

$$
\Delta P\left(k_{1}, k_{2} ; x_{1}, x_{2}, t\right)=2 \tilde{P}\left(k_{1}, k_{2}\right) g\left(x_{1}, k_{1}, k_{2}\right) e^{-i\left[k_{1}\left(x_{1}-U_{c} t\right)+k_{2} x_{2}\right]}
$$

where $\tilde{P}\left(k_{1}, k_{2}\right)$ is the amplitude of the pressure jump. Because of the assumed convective nature of the turbulence, the notation for the chordwise wave number $k_{1}$ will be replaced by $k_{c}$ to emphasize its dependence on the convection speed $U_{c}$. In an exact sense, an infinite number of combinations of frequency and convection speed ratio $\omega / U_{c}$ would yield a given value of $k_{c}=\omega / U_{c}$. However, the acoustically relevant structures in the turbulent boundary layer are assumed frozen with respect to a single convection speed that is taken as $U_{c}=0.8 U$.

The complete broadband surface pressure jump $\Delta P\left(x_{1}, x_{2}, t\right)$ is obtained by summing all Fourier components in Eq. (4):

$$
\Delta P\left(x_{1}, x_{2}, t\right)=2 \int_{-\infty}^{\infty} \int_{-\infty}^{\infty} \tilde{P}\left(k_{c}, k_{2}\right) g\left(x_{1}, k_{c}, k_{2}\right) e^{-i\left[k_{c}\left(x_{1}-U_{c} t\right)+k_{2} x_{2}\right]} d k_{c} d k_{2}
$$

A straightforward approach for predicting the desired broadband far field measurements is to 
use the real part of Eq. (5) as input to Formulation 1B. This approach requires knowledge of a two-component surface pressure spectrum and a dual wave number transfer function $g\left(x_{1}, k_{c}, k_{2}\right)$. However, because one of the objectives of the current work is to reproduce the results of Schlinker and Amiet [16] from a time domain perspective, an approach similar to theirs taken will be used to model the surface pressure.

The analysis for the frequency-domain formulation in [16] comes from previous work [22] in which Amiet argues that only one spanwise wave number contributes to the sound detected by an observer in a given location. In particular, Amiet focuses on an observer in a spanwise symmetric location, for which only the zero spanwise wave number needs to be considered. An analogous timedomain result was derived in [7]. Although the analyses in [22] and [7] were presented to derive an acoustic formulation for incident turbulence noise, the results pertaining to spanwise wave numbers are sufficiently general to apply to the present trailing edge problem, with the only significant difference being the transfer function $g\left(x_{1}, k_{1}, k_{2}\right)$. Therefore, applying the time-domain analysis in [7] to the present problem yields the following simplified expression for the surface pressure:

$$
\begin{aligned}
\Delta P\left(x_{1}, t\right) & =2 \pi \int_{-\infty}^{\infty} \tilde{P}\left(k_{c}, 0\right) g\left(x_{1}, k_{c}, 0\right) e^{-i k_{c}\left(x_{1}-U_{c} t\right)} d k_{c} \\
p(\vec{y}, \tau) & =\Re\left\{-\Delta P\left(y_{1}, \tau\right)\right\}
\end{aligned}
$$

Note that Eq. (6) is valid as an acoustic source pressure for an observer in the plane $x_{2}=0$, and the $k_{2}$ integration in Eq. (5) is neglected. Furthermore, all three integrals in Eq. (1) will be evaluated for the predictions that follow, although the first integral is expected to dominate the signal.

The unsteady surface pressure is numerically evaluated by an approach similar to that in [7]. Eq. (6) can be approximated by a truncated series in which the coefficients are chosen such that the autocorrelation and power spectrum of the series form a Fourier transform pair. This approach is consistent with stochastic modeling theory, e.g., [23]. The unsteady surface pressure jump in Eq. 
(6) is then approximated by

$$
\begin{aligned}
\Delta P\left(x_{1}, t\right) & \approx 2 \pi \sum_{n=-N}^{N} A_{n} e^{i \phi_{n}} g\left(x_{1}, k_{c, n}, 0\right) e^{-i k_{c, n}\left(x_{1}-U_{c} t\right)} \\
k_{c, n} & =n \Delta k_{c}, \quad n=0, \pm 1, \pm 2, \ldots, \pm N \\
\Delta k_{c} & =k_{c, N} / N
\end{aligned}
$$

The discrete surface pressure amplitudes $\left\{A_{n}\right\}$ are evaluated by

$$
A_{n}=\left[\Phi_{P P}\left(k_{c, n}, 0\right) \Delta k_{c}\right]^{\frac{1}{2}}
$$

where $\Phi_{P P}\left(k_{c}, k_{2}\right)$ is the two-component power spectral density (PSD) of the surface pressure. Schlinker and Amiet [16] argue that the required single wave number spectrum $\Phi_{P P}\left(k_{c}, 0\right)$ can be evaluated by

$$
\Phi_{P P}\left(k_{c}, 0\right)=\frac{U_{c}}{\pi} \ell_{2}(\omega) S_{q q}(\omega, 0)
$$

where $\ell_{2}(\omega)$ is the spanwise correlation length and $S_{q q}\left(\omega, \Delta x_{2}\right)$ is the spanwise surface pressure correlation function.

The phase angles $\left\{\phi_{n}\right\}$ are independent random variables uniformly distributed on $[0,2 \pi]$. The transfer function in Eq. (3b) can be used for $g\left(y_{1}, k_{c, n}, 0\right)$ with the following modification. As previously noted, Eqs. (3a) and (3b) represent the induced pressure jump. Amiet [5] has suggested that the incident pressure, i.e., the pressure that results from turbulent eddies contacting the trailing edge, can be accounted for by the addition of an exponential convergence factor of the form $e^{\epsilon k_{c} x_{1}}$, where $\epsilon$ is a positive parameter. For $-C \leq x_{1} \leq 0$, this additional term will be significantly larger than zero only in the immediate vicinity of the trailing edge, provided that $\epsilon k_{c} C$ is large. Therefore, to include the effect of the incident pressure, the transfer function to be in Eq. (7a) is the two-component function $g\left(x_{1}, k_{c}, k_{2}\right)$ in [16], with $k_{2}=0$ :

$$
g\left(x_{1}, k_{c}, 0\right)=e^{\epsilon k_{c} x_{1}}-1+(1+i) E^{*}\left[-x_{1}\left(k_{c}+\mu(1+M)\right)\right]
$$


where $E^{*}$ is the same complex combination of Fresnel integrals as in Eq. (3c). Amiet [5] was able to avoid the direct use of the parameter $\epsilon$ because of the manner in which the transfer function in Eq. (7d) was used in his analysis. Amiet used the transfer function to define an unsteady lift response function that involved the chordwise integration of the transfer function with other terms. This integration yields an expression that, after clever manipulation of limits, does not contain $\epsilon$ but still provides an additional term to the lift response function that accounts for the incident pressure. In the present case, the transfer function in Eq. (7d) must be explicitly used and therefore a value for $\epsilon$ must be specified; a value is specified in the following subsection.

To illustrate the effect of including this incident pressure term in the surface pressure formulation, Fig. 8 shows the same single frequency surface pressure fig. 3, with and without the the incident pressure term, with $\epsilon=1$. Clearly, the incident pressure term has a significant effect only near the trailing edge, as expected. However, for a given frequency, differing values of $\epsilon$ will affect differing amounts of upstream chordlength. Note that the addition of this term causes the pressure jump to vanish at the trailing edge for all time, i.e., the Kutta condition is satisfied. Note also the increase in spatial oscillation near the trailing edge when this incident pressure term is included.

Eqs. (7a)-(7d) represent the the complex-valued broadband surface pressure formulation used for the present TE noise predictions. The final representation for the unsteady broadband pressure on the airfoil surface is then given by the real part of $-\Delta P$ in Eq. (7a). Using symmetry arguments and algebraic manipulation, the indicial bounds for the surface pressure spectral representation are altered so that the domain includes only positive wave numbers. As input to Eq. (1), the resulting real-valued surface pressure can be written

$$
p\left(y_{1}, \tau\right)=-4 \pi \sum_{n=1}^{N} A_{n}\left\{B_{n} \cos \left[k_{c, n}\left(y_{1}-U_{c} \tau\right)+\phi_{n}\right]+D_{n} \sin \left[k_{c, n}\left(y_{1}-U_{c} \tau\right)+\phi_{n}\right]\right\}
$$


where

$$
\begin{aligned}
A_{n} & =\left[\frac{U_{c}}{\pi} \ell_{2}\left(\omega_{n}\right) S_{q q}\left(\omega_{n}, 0\right) \Delta k_{c}\right]^{\frac{1}{2}} \\
B_{n} & =e^{\epsilon k_{c, n} y_{1}}-1+\mathcal{C}\left(\xi_{n}\right)+\mathcal{S}\left(\xi_{n}\right) \\
D_{n} & =\mathcal{C}\left(\xi_{n}\right)-\mathcal{S}\left(\xi_{n}\right) \\
\xi_{n} & =-y_{1}\left[k_{c, n}+\mu_{n}(1+M)\right]
\end{aligned}
$$

and $\mathcal{C}\left(\xi_{n}\right)$ and $\mathcal{S}\left(\xi_{n}\right)$ are the Fresnel cosine and sine integrals in Eq. (3c). Specific evaluations for the correlation lengths $\ell_{2}$ and surface pressure correlations $S_{q q}$ will be discussed in the following subsection. Furthermore, the zeroth term in Eq. (8a) is omitted because of the singularity in the formulation for the correlation length at $n=0$.

\subsection{TIME DOMAIN PREDICTIONS}

The lower and upper frequency bounds for both calculations are $25 \mathrm{~Hz}$ and $10 \mathrm{kHz}$, respectively. Therefore, $f=25 \mathrm{~Hz}$ also serves as the fundamental frequency and the numerical bandwidth $\Delta f$. Each calculation is performed for one period of the lowest frequency, $T=0.04$ sec. The numerical solution is sampled at the Nyquist frequency, i.e., $\Delta t=T / 2 N$. The calculation is performed on a $500 \times 100$ surface grid with grid-point clustering near the trailing edge, as shown in Fig. 9. This trailing edge clustering allows for better resolution to account for the effect of the incident pressure term in Eq. (7d), as previously shown in Fig. 8. As in the constant frequency case, because the surface pressure in Eqs. (8a)-(8e) is cast in only one spatial variable $y_{1}$, and the observer location is symmetric relative to the airfoil span, the acoustic predictions are found to be relatively insensitive to the discretization in $y_{2}$; the primary concern for grid resolution is in the streamwise direction. With 500 points in the streamwise direction and clustering near the trailing edge, a sufficient resolution of at least 10 points per wavelength was obtained for the entire length of the chord. This conclusion was reached by inspecting surface pressure profiles for the highest frequency of $10 \mathrm{kHz}$.

The coordinate system for the calculation is such that the $x_{2}$-axis coincides with the center span 
line, and the microphone position is in the plane $x_{2}=0$. The experimental microphone position for which comparisons are made is at a distance of $1.22 \mathrm{~m}$ from the model, at an angle of 90 degrees relative to the chord and directly above the trailing edge. Thus, the measured observer position for the prediction is, $\vec{x}=[0,0,1.22]^{T}$ in meters.

The baseline prediction case under consideration is for a tunnel speed of $U=69.5 \mathrm{~m} / \mathrm{s}$. This flow condition, the above observer location, and airfoil geometry are incorporated into an acoustic prediction using Eq. (1) with the surface pressure defined by Eqs. (8a)-(8e). Following the example of Schlinker and Amiet [16], a first-cut prediction is performed by using flat plate theory to evaluate the surface pressure correlations $S_{q q}(\omega, 0)$ and spanwise correlation lengths $\ell_{2}(\omega)$ in Eq. (8b). Schlinker and Amiet used empirical formulations for these quantities that they derived from previous analysis and boundary layer measurements of Corcos [13] and Willmarth and Roos [14]. The surface pressure correlations are approximated by

$$
S_{q q}(\omega, 0) \approx q_{0}^{2} \frac{\delta^{*}}{U} \frac{2 \times 10^{-5}}{1+\tilde{\omega}+0.217 \tilde{\omega}^{2}+0.00562 \tilde{\omega}^{4}}
$$

where $q_{0}=\rho_{0} U^{2} / 2, \delta^{*}$ is the trailing edge displacement thickness, and $\tilde{\omega}=\omega \delta^{*} / U$. The displacement thickness is also taken from a flat plate approximation for turbulent boundary layer thickness $\delta$ on a flat plate, based on the chord Reynolds number $R e_{C}$, i.e.,

$$
\frac{\delta}{C} \approx \frac{0.37}{\left(R e_{C}\right)^{\frac{1}{5}}} \approx 8 \frac{\delta^{*}}{C}
$$

For the experiment of Brooks and Hodgson [17], Schlinker and Amiet [16] used Eq. (9b) to compute the boundary layer thickness $\delta$ and accounted for boundary layer tripping by taking the 15 percent chord station as the initial point of the calculation. Surface curvature was also accounted for in the downstream distance used in the calculation. The ratio $\delta / C$ used by Schlinker and Amiet for this experiment was reported as 0.0166 for $U=69.5 \mathrm{~m} / \mathrm{s}$ and 0.0187 for $U=38.6 \mathrm{~m} / \mathrm{s}$. The displacement thickness was then taken as $1 / 8$ of the boundary layer thickness. The expression that 
Schlinker and Amiet [16] suggest for the spanwise correlation length is

$$
\ell_{2}(\omega) \approx \frac{2.1 U_{c}}{\omega}
$$

Fig. 10 shows the far field signal $p^{\prime}(\vec{x}, t)$ that is predicted by Formulation $1 \mathrm{~B}$ at the experimental microphone location, for a tunnel speed of $69.5 \mathrm{~m} / \mathrm{s}$. The surface pressure is modeled with Eqs. $(8 \mathrm{a})-(8 \mathrm{e})$ and $(9 \mathrm{a})-(9 \mathrm{c})$. The time signal $p^{\prime}(\vec{x}, t)$ is Fourier analyzed to determine a discrete set of spectral amplitudes $\left\{P_{n}\right\}_{n=1}^{N}$. The far field sound pressure level (SPL) spectrum is calculated by

$$
S P L\left(f_{n}\right)=20 \log \left[\frac{P_{n}}{P_{\text {ref }}}\right], \quad n=1,2, \ldots, N
$$

where the reference pressure is $P_{\text {ref }}=20 \mu \mathrm{Pa}$. The SPLs are converted to a $1.0 \mathrm{~Hz}$ bandwidth by reducing the values in Eq. (10) by $10 \log (\Delta f)$.

The resulting narrowband SPLs are compared with the prediction of Schlinker and Amiet [16] in Fig. 11. Also on this plot are the narrowband SPLs that were experimentally measured by Brooks and Hodgson [17]. The predicted results of Schlinker and Amiet and the measurements of Brooks and Hodgson shown in Fig. 11 were obtained by digitizing the appropriate plots of Schlinker and Amiet (Figure 34 of Reference 16). Various values of the parameter $\epsilon$ in Eq. (7d) were assessed in this comparison stage of the research. The value $\epsilon=1.5$ was chosen for its agreement with the flat plate correlation results of Schlinker and Amiet [16]. This value of $\epsilon$ is held fixed for the remaining calculations.

Clearly, Fig. 11 shows that significant error exists between the predictions and the measurements when flat plate formulations are used for the required surface pressure correlations. Fig. 12 sheds light on this error by comparing the flat plate formula in Eq. (9a) with the measured surface pressure correlations of Yu and Joshi [15]. The notation $\bar{S}_{q q}$ denotes that the surface pressure correlations are normalized by $q_{0}^{2} \delta^{*} / U$. The measured data in Fig. 12 were obtained by digitizing the "average" correlation plot of Schlinker and Amiet (Figure 35(a) of Reference 16). The normalized flat plate surface pressure correlations are significantly lower than the measured data, by as much as $7 \mathrm{~dB}$. 
The significant error in the flat plate approximation is only partially due the the lack of pressure gradient. The more significant error made in the approximation in Eq. (9a) is the lack of a trailing edge in its derivation. This empirical formulation is based on experimental measurement and analysis in which the flat plate is assumed to be infinite. Clearly, surface pressure correlations that are based on flat plate theory are inappropriate for predicting TE noise in this case.

The experimental surface pressure correlations in Fig. 12 will now be used in the surface pressure formulation to predict the TE noise associated with the two tunnel speeds of interest and compared with experimental measurements. The modified formula for the surface pressure correlations is

$$
S_{q q}(\omega, 0) \approx q_{0}^{2} \frac{\delta^{*}}{U} \bar{S}_{q q}(\omega, 0)
$$

where $\bar{S}_{q q}(\omega, 0)$ denotes the normalized measured data in Fig. 12. The tabulated data for this measured surface pressure correlation function is stored in a file that can be accessed and interpolated

to obtain $\bar{S}_{q q}(\omega, 0)$ for any frequency. The alteration of the surface pressure correlation function by experimental data now brings into question the evaluation of the spanwise correlation length. However, it was concluded by Brooks and Hodgson [17] that the function $\ell_{2}(\omega)$ for a flat plate and a thin airfoil are identical under suitable normalization. Therefore, the use of Eq. (9c) for $\ell_{2}(\omega)$ will be retained for the remaining calculations.

The predicted and measured far field SPLs for the two tunnel speeds are shown in Fig. 13. The experimental data in Fig. 13 were obtained by digitizing the measurements plotted in Figure 34 of Reference 16 . The agreement with the measured data is significantly improved when the calculation includes surface pressure correlations that account for the trailing edge of an airfoil. In fact, it was concluded by Schlinker and Amiet [16] that airfoil surface pressure correlations were absolutely necessary for realistic TE noise predictions.

\section{CONCLUDING REMARKS}

The prediction of broadband trailing edge noise from rotating machinery and airframes is cur- 
rently the subject of intense research in aeroacoustics. The physics of broadband noise generation are well understood as the result of the pioneering research of Howe [1, 24, 25], Amiet and coworkers $[4,5,12,16,22]$, and Brooks and coworkers $[17,26]$. The previous work of these and many other aeroacousticians has clearly demonstrated that any successful broadband loading noise prediction requires an understanding of two physical processes: the character of the time-dependent surface pressure that provides the acoustic source, and the manner in which that source gives rise to an acoustic signal.

Obtaining the fluctuating surface pressure distribution analytically, numerically, or experimentally is itself a difficult problem. For this reason, past researchers have most often resorted to modeling the surface pressure, using guidance from experiments. However, as computational technology continues to advance, high resolution surface pressure fluctuations could soon become available from simulations of realistic problems. Therefore, the improvement of the acoustic radiation model becomes an important research topic.

In the present work, a a loading-noise solution of the Ffowcs Williams-Hawkings equation is applied to the prediction of broadband trailing edge noise on a NACA 0012 airfoil in a low Mach number flow. This new solution, originally proposed in [7], is to date the simplest analytical result for the prediction of loading noise and is suitable for statistical analysis of broadband noise for a surface in general motion. The time domain predictions are found to be in excellent agreement with the frequency domain predictions of Schlinker and Amiet [16] as well as with the experimental measurements of Brooks and Hodgson [17]. These results are, to the authors' knowledge, the first successful broadband trailing edge noise predictions in the time domain.

\section{ACKNOWLEDGMENT}

The authors would like to express their gratitude to Dr. Roy K. Amiet, for his valuable input from his past experience. 


\section{REFERENCES}

1. M. S. Howe 1978 Journal of Sound and Vibration, Vol. 61, pp. 437-465. A Review of the Theory of Trailing Edge Noise.

2. M. J. Lighthill 1952 Proceedings of the Royal Society of London, A 211, pp. 564-587. On Sound Generated Aerodynamically. I. General Theory.

3. J. E. Ffowcs Williams and L. H. Hall 1970 Journal of Fluid Mechanics, Vol. 40, pp. 657-670. Aerodynamic Sound Generation by Turbulent Flow in the Vicinity of a Scattering Half-Plane.

4. R. K. Amiet 1976 Journal of Sound and Vibration, Vol. 47, No. 3, pp. 387-393. Noise Due to a Turbulent Flow Past a Trailing Edge.

5. R. K. Амiet 1978 Journal of Sound and Vibration, Vol. 57, No. 2, pp. 305-306. Effect of the Incident Surface Pressure Field on Noise Due to a Turbulent Flow Past a Trailing Edge.

6. M. E. Goldstein 1979 Journal of Fluid Mechanics, Vol. 91, No. 4, pp. 601-632. Scattering and Distortion of the Unsteady Motion on Transversely Sheared Mean Flows.

7. J. Casper and F. Farassat 2002 International Journal of Aeroacoustics, Vol. 1, No. 3, pp. 207-240. A New Time Domain Formulation for Broadband Noise Predictions.

8. J. E. Ffowcs Williams and D. L. Hawkings 1969 Philosophical Transactions of the Royal Society, A 264, pp. 321-342. Sound Generation by Turbulence and Surfaces in Arbitrary Motion.

9. B. A. Singer, K. S. Brentner, D. P. Lockard, and G. M. Lilley 2000 Journal of Sound and Vibration, Vol. 230, No. 3, pp. 541-560. Simulation of Acoustic Scattering from a Trailing Edge. doi:10.1006/jsvi.1999.2628

10. F. Farassat and G. P. Succi 1983 Vertica, Vol. 7, No. 4, pp. 309-320. The Prediction of Helicopter Rotor Discrete Frequency Noise. 
11. R. W. Paterson and R. K. Amiet 1977 AIA A Journal of Aircraft, Vol. 14, No. 8, pp. 729-736. Noise and Surface Pressure Response of an Airfoil to Incident Turbulence.

12. R. K. Amiet 1976 AIAA Journal, Vol. 14, No. 8, pp. 1076-1082. High Frequency Thin-Airfoil Theory for Subsonic Flow.

13. G. M. Concos 1964 Journal of Fluid Mechanics, Vol. 18, pp. 353-378. The Structure of the Turbulent Pressure Field in Boundary Layer Flows.

14. W. W. Willmarth and F. W. Roos 1965 Journal of Fluid Mechanics, Vol. 22, pp. 81-94. Resolution and Structure of the Wall Pressure Field Beneath a Turbulent Boundary Layer.

15. J. C. YU and M. C. Joshi 1979 AIAA Paper No. 79-0603. On Sound Radiation from the Trailing Edge of an Isolated Airfoil in a Uniform Flow.

16. R. H. SchlinkeR and R. K. Amiet 1981 NASA Contractor Report No. 3470. Helicopter Rotor Trailing Edge Noise.

17. T. F. Brooks and T. H. Hodgson 1981 Journal of Sound and Vibration, Vol. 78, No. 1, pp. 69-117. Trailing Edge Noise Prediction from Measured Surface Pressures.

18. J. Boersma 1960 Mathematics of Computation, Vol. 14, p. 380. Computation of Fresnel Integrals.

19. N. CurLe 1954 Proceedings of the Royal Society of London, A 231, pp. 505-514. The Influence of Solid Boundaries on Aerodynamic Sound.

20. W. G. Halvorsen and J. S. Bendat 1975 Journal of Sound and Vibration, Vol. 9, pp. 15-24. Noise Source Identification Using Coherent Output Power Spectra.

21. A. G. Piersol 1978 Journal of Sound and Vibration, Vol. 56, pp. 215-228. Use of Coherence and Phase Data Between Two Receivers in Evaluation of Noise Environments.

22. R. K. Amiet 1975 Journal of Sound and Vibration, Vol. 41, pp. 407-420. Acoustic Radiation from an Airfoil in a Turbulent Stream. 
23. M. Shinozuka and G. Deodatis 1991 Applied Mechanics Review, Vol. 44, No. 4, pp. 191-204. Simulation of Stochastic Processes by Spectral Representation.

24. M. S. Howe 1999 Journal of Sound and Vibration, Vol. 225, No. 2, pp. 211-238. Trailing Edge Noise at Low Mach Numbers.

25. M. S. Howe 2000 Journal of Sound and Vibration, Vol. 234, No. 5, pp. 761-775. Trailing Edge Noise at Low Mach Numbers, Part 2: Attached and Separated Flows.

26. T. F. Brooks, D. S. Pope, and A. M. Marcolini 1989 NASA Reference Publication No. 1218. Airfoil Self-Noise and Prediction. 


\section{APPENDIX: LIST OF SYMBOLS}

$$
\begin{aligned}
& b=\text { airfoil semi-span (m) } \\
& C=\text { airfoil chord (m) } \\
& c_{0}=\text { ambient sound speed }(\mathrm{m} / \mathrm{sec}) \\
& f=\text { frequency }(\mathrm{Hz}) \\
& \tilde{f}=\text { geometry function for airfoil surface (Fig. 1) } \\
& E^{*}=\text { combination of Fresnel integrals (Eq. (3c)) } \\
& g=\text { surface pressure transfer function } \\
& k_{c}=\omega / U_{c} \text {, convective wave number }(\mathrm{rad} / \mathrm{m}) \\
& \ell_{2}=\text { spanwise correlation length }(\mathrm{m}) \\
& \vec{M}=\vec{V} / c_{0} \text {, Mach number vector } \\
& M_{r}=\vec{M} \cdot \vec{r} / r \text {, Mach number in radiation direction } \\
& M_{\nu}=\vec{M} \cdot \hat{\nu} \text {, Mach number in direction of } \hat{\nu} \\
& \Delta P=\text { unsteady airfoil surface pressure jump (Pa) } \\
& p=\text { unsteady airfoil surface pressure }(\mathrm{Pa}) \\
& p^{\prime}=\text { sound pressure radiated to observer }(\mathrm{Pa}) \\
& \partial p / \partial s=\text { surface pressure gradient in the direction of } \vec{V}(\mathrm{~Pa} / \mathrm{m}) \\
& q_{0}=\rho_{0} U^{2} / 2 \text {, dynamic head }(\mathrm{Pa}) \\
& \vec{r}=\vec{x}-\vec{y}, \text { sound radiation vector }(\mathrm{m}) \\
& S_{q q}=\text { surface pressure correlation function }\left(\mathrm{Pa}^{2}-\mathrm{sec} / \mathrm{rad}^{2}\right) \\
& T=1 / f, \text { acoustic period }(\mathrm{sec}) \\
& t=\text { observer time (sec) } \\
& U=\text { uniform freestream speed }(\mathrm{m} / \mathrm{sec}) \\
& \vec{V}=\text { airfoil velocity vector }(\mathrm{m} / \mathrm{sec}) \\
& \vec{x}=\left[x_{1}, x_{2}, x_{3}\right]^{T} \text {, observer position (m) } \\
& \vec{y}=\left[y_{1}, y_{2}, 0\right]^{T} \text {, surface source position (m) }
\end{aligned}
$$




$$
\begin{aligned}
\beta & =\sqrt{1-M^{2}} \\
\delta & =\text { boundary layer thickness } \\
\delta^{\star} & =\text { displacement thickness } \\
\lambda & =c_{0} / f, \text { acoustic wave-length }(\mathrm{m}) \\
\mu & =M \omega / \beta^{2} U(\mathrm{rad} / \mathrm{m}) \\
\psi & =\text { directivity angle }(\text { Fig. } 5)(\mathrm{rad}) \\
\hat{\nu} & =\text { unit inward facing normal on surface edge }(\mathrm{Fig} .1) \\
\theta & =\text { angle between surface normal and } \vec{r}(\text { Fig. } 1)(\mathrm{rad}) \\
\rho_{0} & =\text { ambient density }\left(\mathrm{kg} / \mathrm{m}^{3}\right) \\
\tau & =\text { source time }(\text { sec }) \\
\Phi_{P P} & =\text { power spectral density of surface pressure }\left(\mathrm{Pa}^{2}-\mathrm{m}^{2} / \mathrm{rad}^{2}\right) \\
\phi & =\text { random phase variable (rad) } \\
\omega & =2 \pi f, \text { circular frequency }(\mathrm{rad} / \text { sec })
\end{aligned}
$$

\section{Subscripts}

1,2,3 = Cartesian coordinate directions

ret $=$ evaluated at retarded time $t-r / c_{0}$ 


\section{LIST OF FIGURES}

1. Schematic for Formulation 1B derivation.

2. Schematic for the constant-frequency trailing edge noise problem in Section 3.

3. Initial surface pressure profile using Eqs. (3a)-(3d).

4. Schematic for directivity calculation, observer on circular path in plane $x_{2}=0$.

5. Directivity for a constant frequency source of $2.5 \mathrm{kHz}$; observer distance $2 \mathrm{~m}$; spacing between concentric circles on grid represents $0.25 \mathrm{~Pa}$.

6. Velocity scaling properties as determined by Formulation 1B and the surface pressure in Eqs. (3a) $-(3 \mathrm{~d}) . \quad-$ - , prediction; ---, slope $=5$.

7. Schematic for trailing edge noise experiment of Brooks and Hodgson [17]

8. Effect of incident pressure term on initial surface pressure profile using Eqs. (3a)-(3d) and modified with Eq. (7d). - - - , induced pressure; - - , sum of induced and incident pressure.

9. Surface grid for prediction of experiment of Brooks and Hodgson [17]; every fourth point in each direction is shown.

10. Predicted far field signal, $U=69.5 \mathrm{~m} / \mathrm{s} ;$ microphone at $90^{\circ}, 1.22 \mathrm{~m}$ above trailing edge.

11. Predicted and measured far field noise spectra; predictions performed with surface pressure correlations from flat plate theory; $U=69.5 \mathrm{~m} / \mathrm{s} . \quad \bigcirc$, frequency domain prediction from [16]; - , time domain domain prediction; - - - experimental measurement [17].

12. Normalized surface pressure correlations. — , from flat plate theory (Eq. (9a)); $-\bigcirc-$, experimental measurements from [15].

13. Predicted and measured far field noise spectra; predictions derived from time domain formulation (Eq. 6) and measured surface pressure correlations [15]. $\triangle$, prediction for $U=38.6 \mathrm{~m} / \mathrm{s} ; \quad \bigcirc$, prediction for $U=69.5 \mathrm{~m} / \mathrm{s} ; \longrightarrow$, experimental SPLs from [17]. 


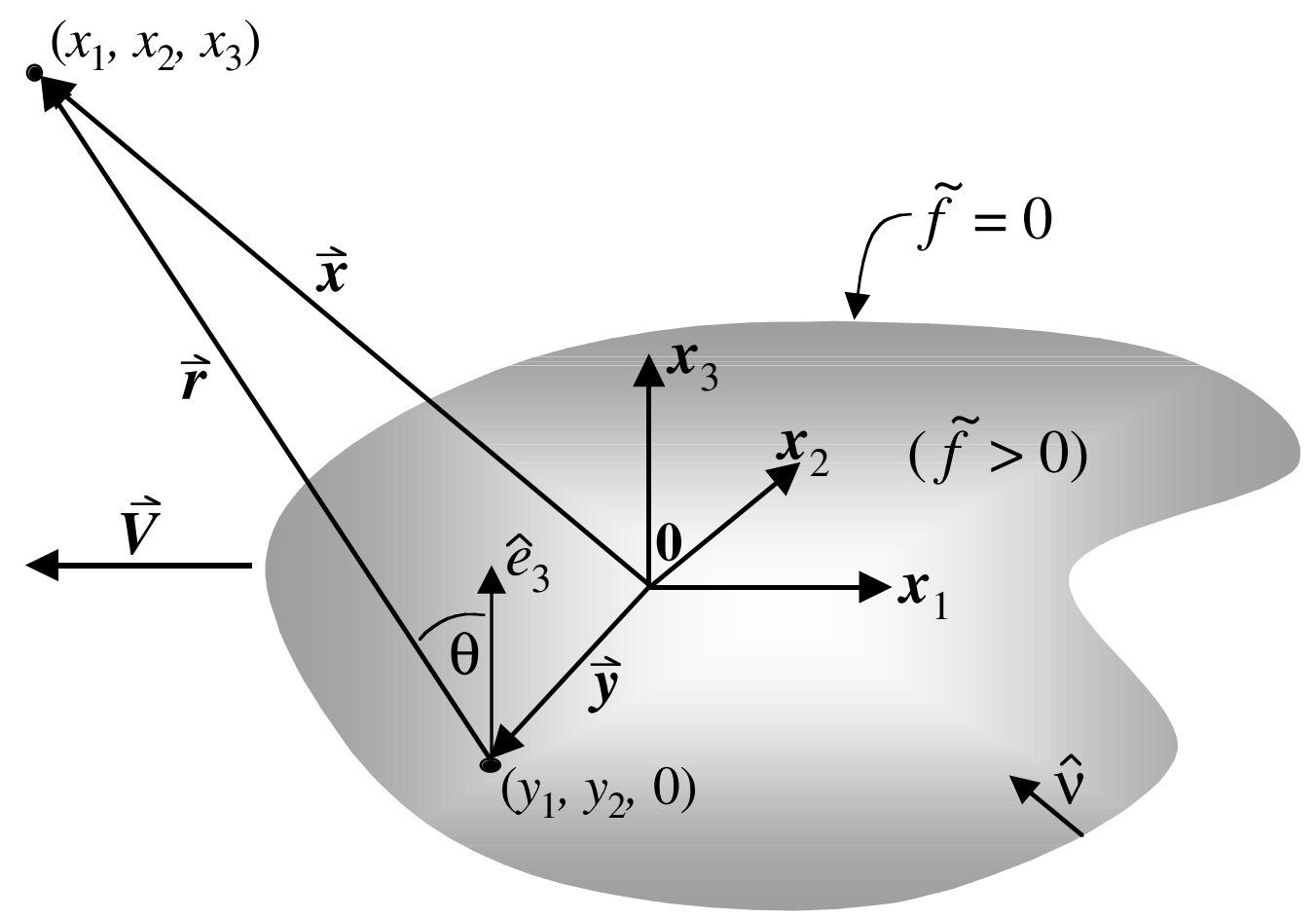

Figure 1 Schematic for Formulation 1B derivation. 


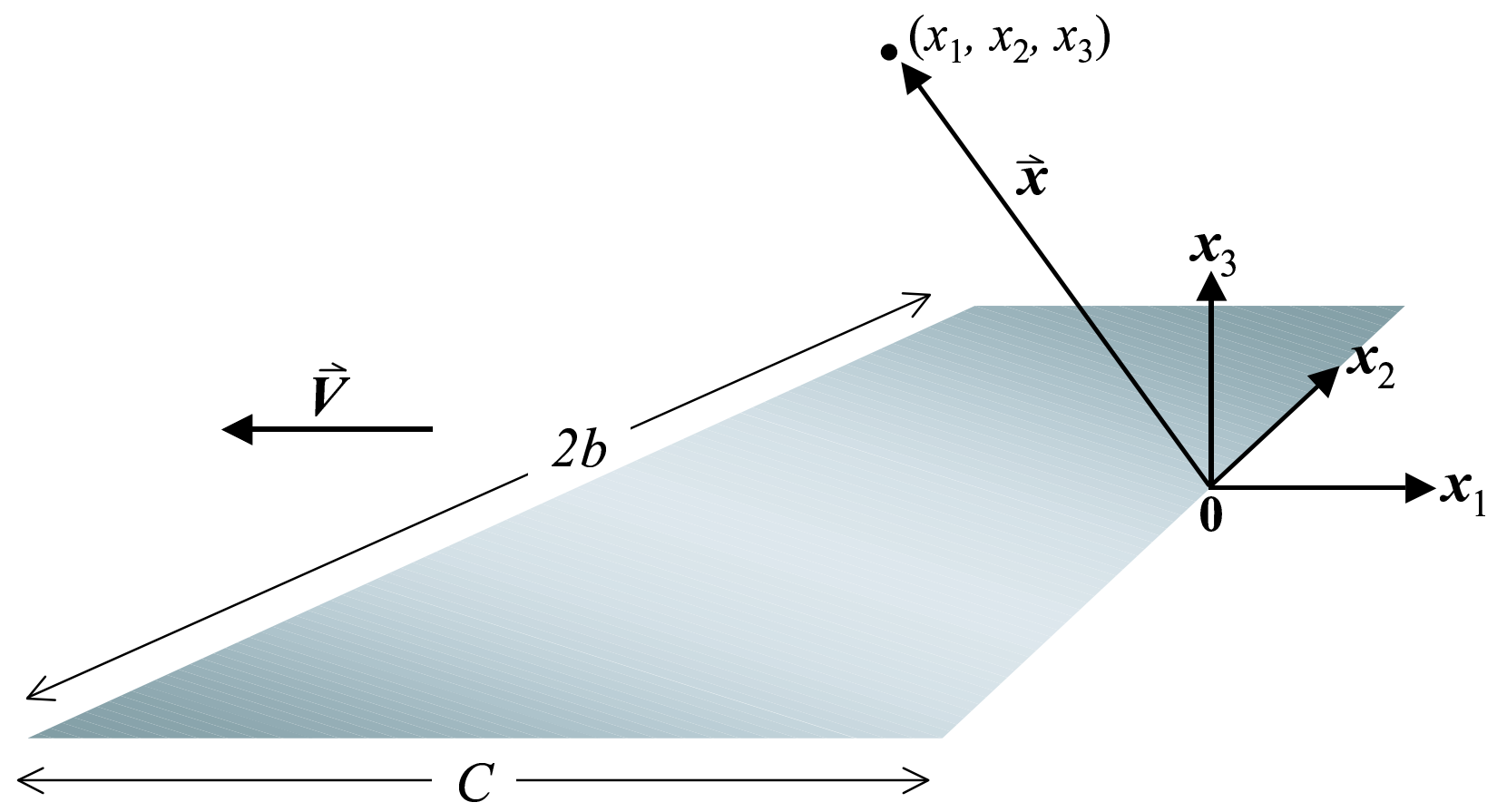

Figure 2 Schematic for the constant-frequency trailing edge noise problem in Section 3. 


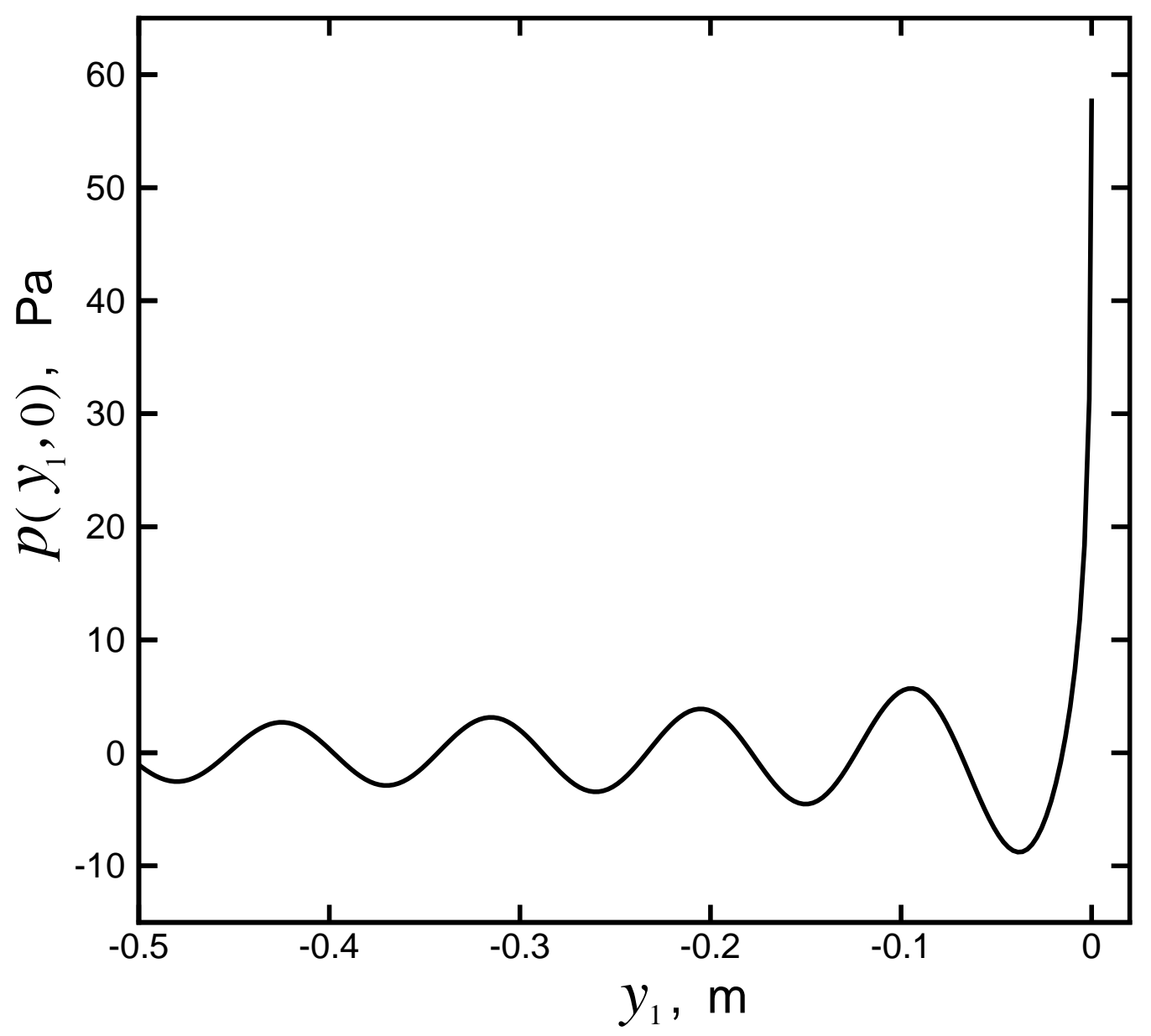

Figure 3 Initial surface pressure profile using Eqs. (3a)-(3d). 


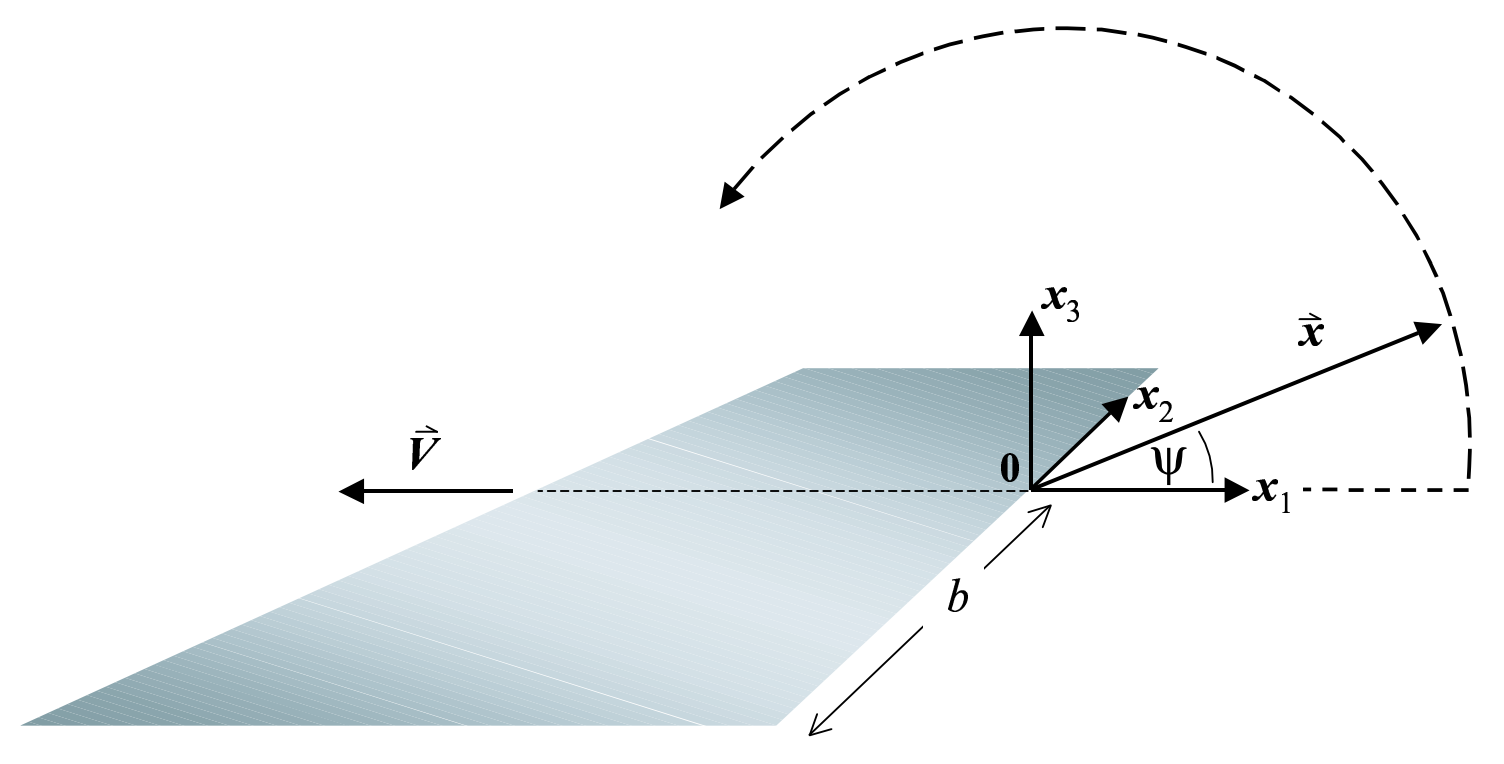

Figure 4 Schematic for directivity calculation, observer on circular path in plane $x_{2}=0$. 


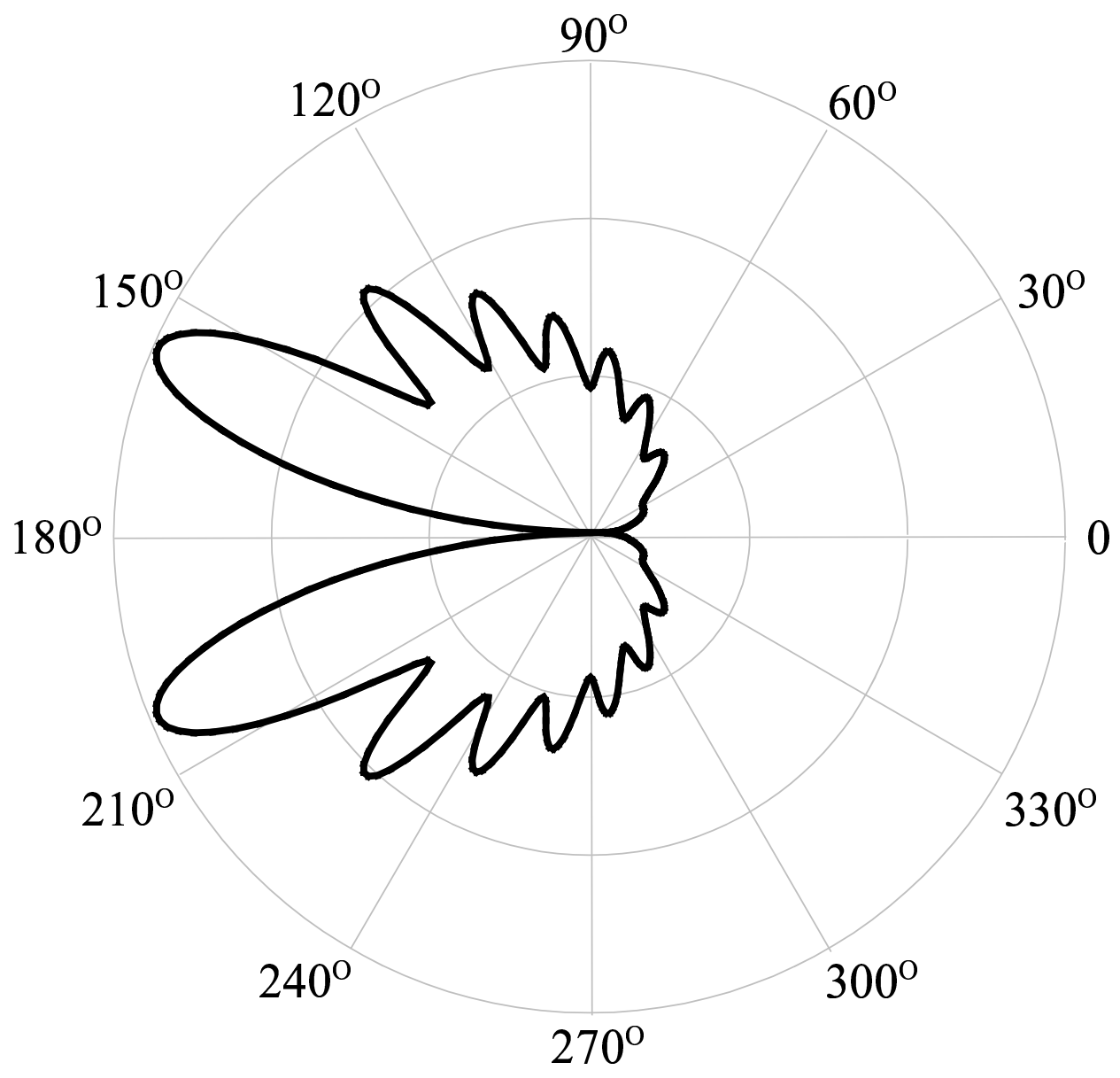

Figure 5 Directivity for a constant frequency source of $2.5 \mathrm{kHz}$; observer distance $2 \mathrm{~m}$; spacing between concentric circles on grid represents $0.25 \mathrm{~Pa}$. 


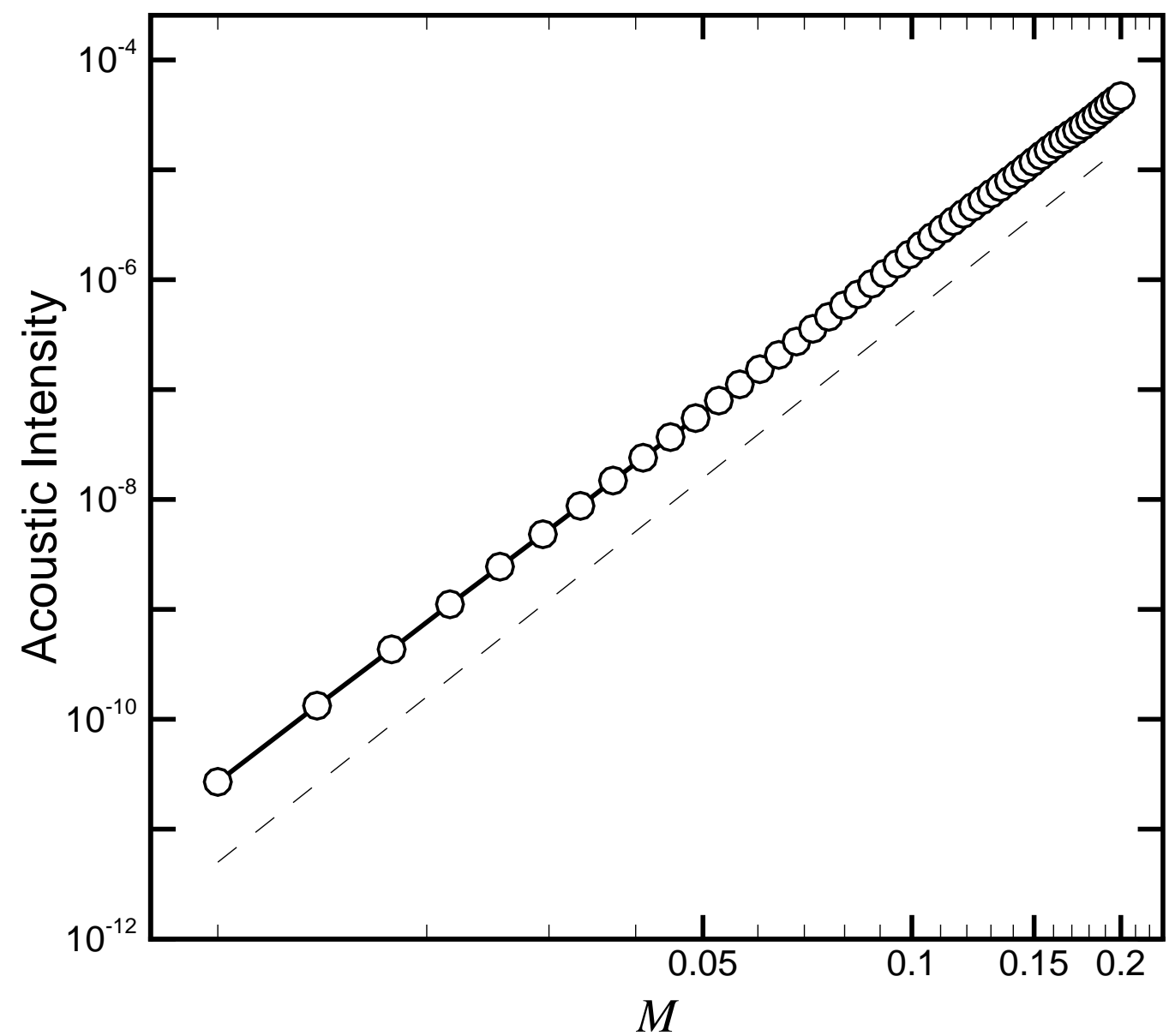

Figure 6 Velocity scaling properties as determined by Formulation $1 \mathrm{~B}$ and the surface pressure in Eqs. (3a)-(3d). $-\bigcirc$, prediction; --- , slope $=5$. 


\section{microphone \{}

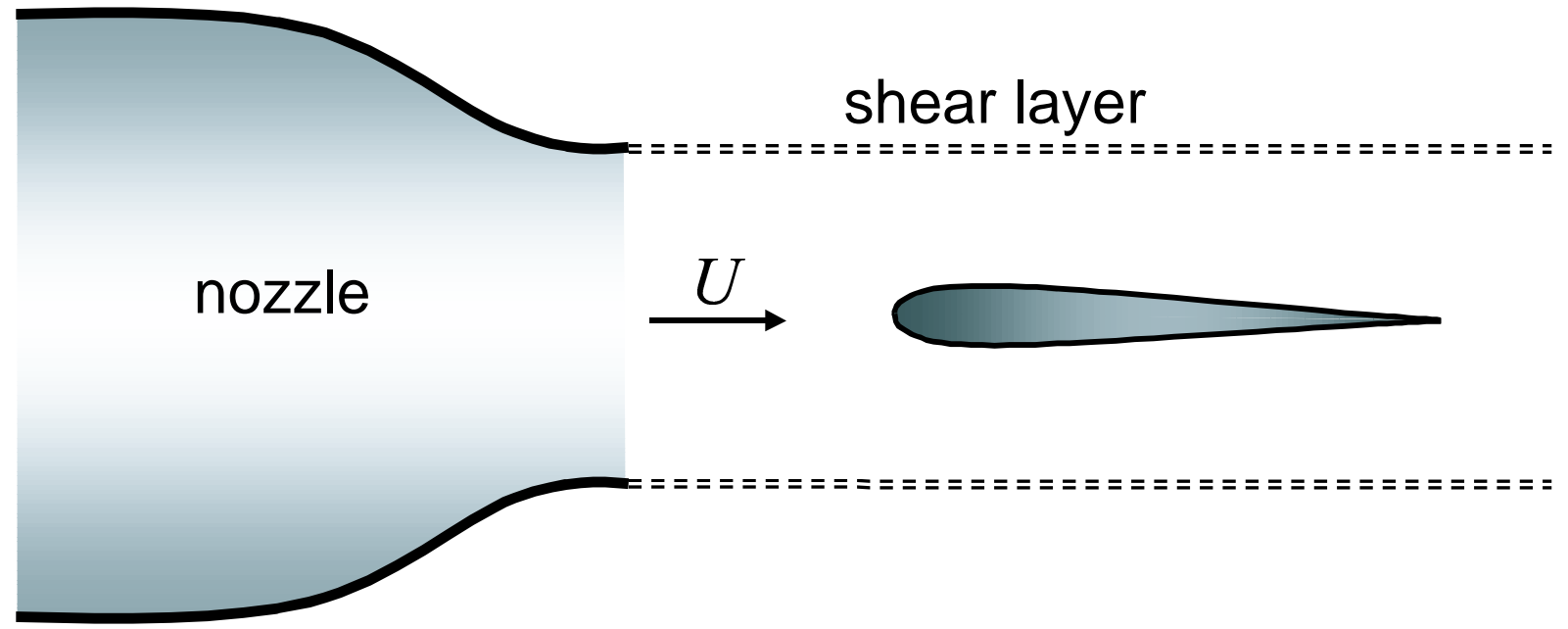

Figure 7 Schematic for trailing edge noise experiment of Brooks and Hodgson [17]. 


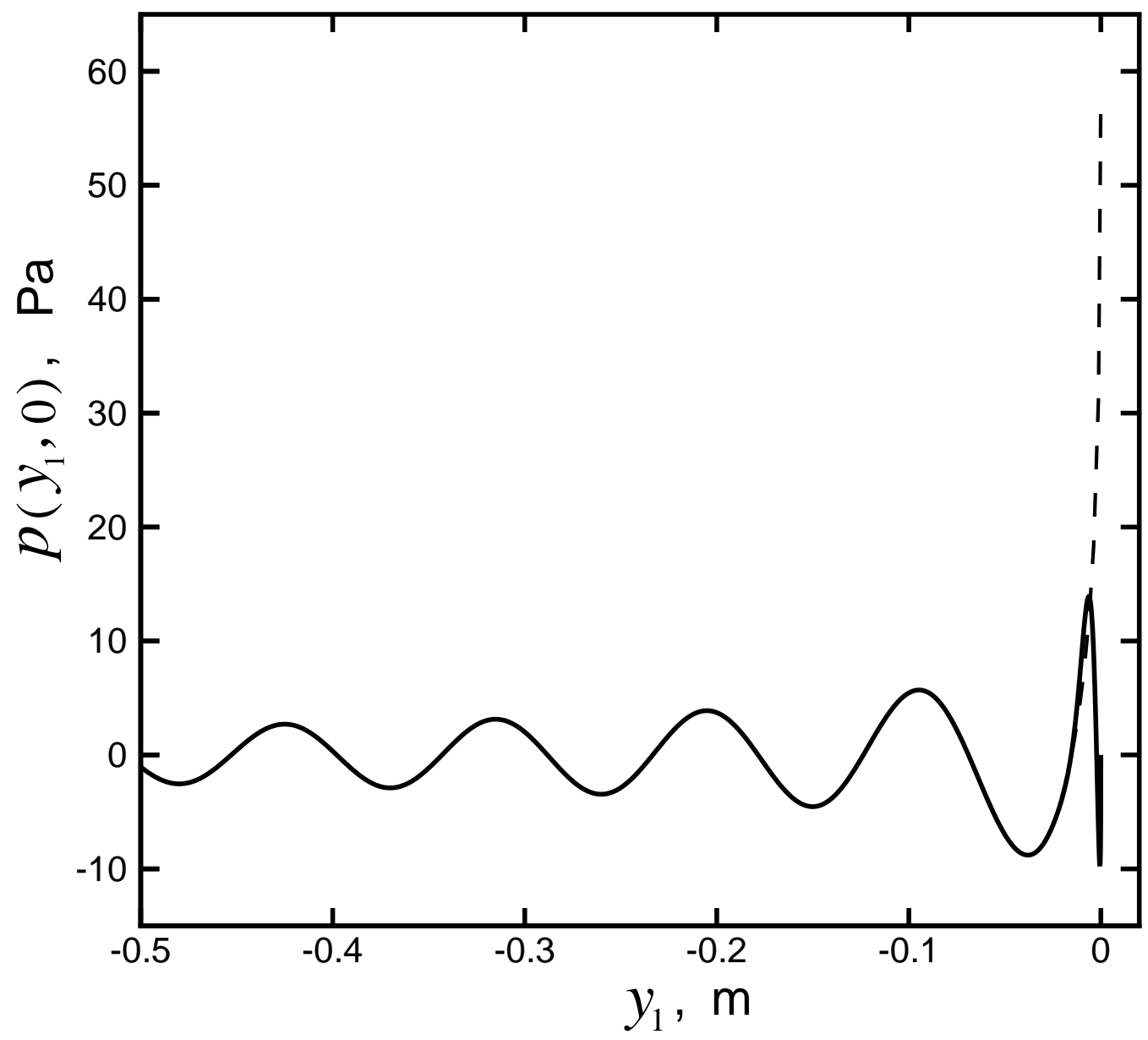

Figure 8 Effect of incident pressure term on initial surface pressure profile using Eqs. (3a)-(3d), and modified with Eq. (7d). --- , induced pressure; —_, sum of induced and incident pressure. 


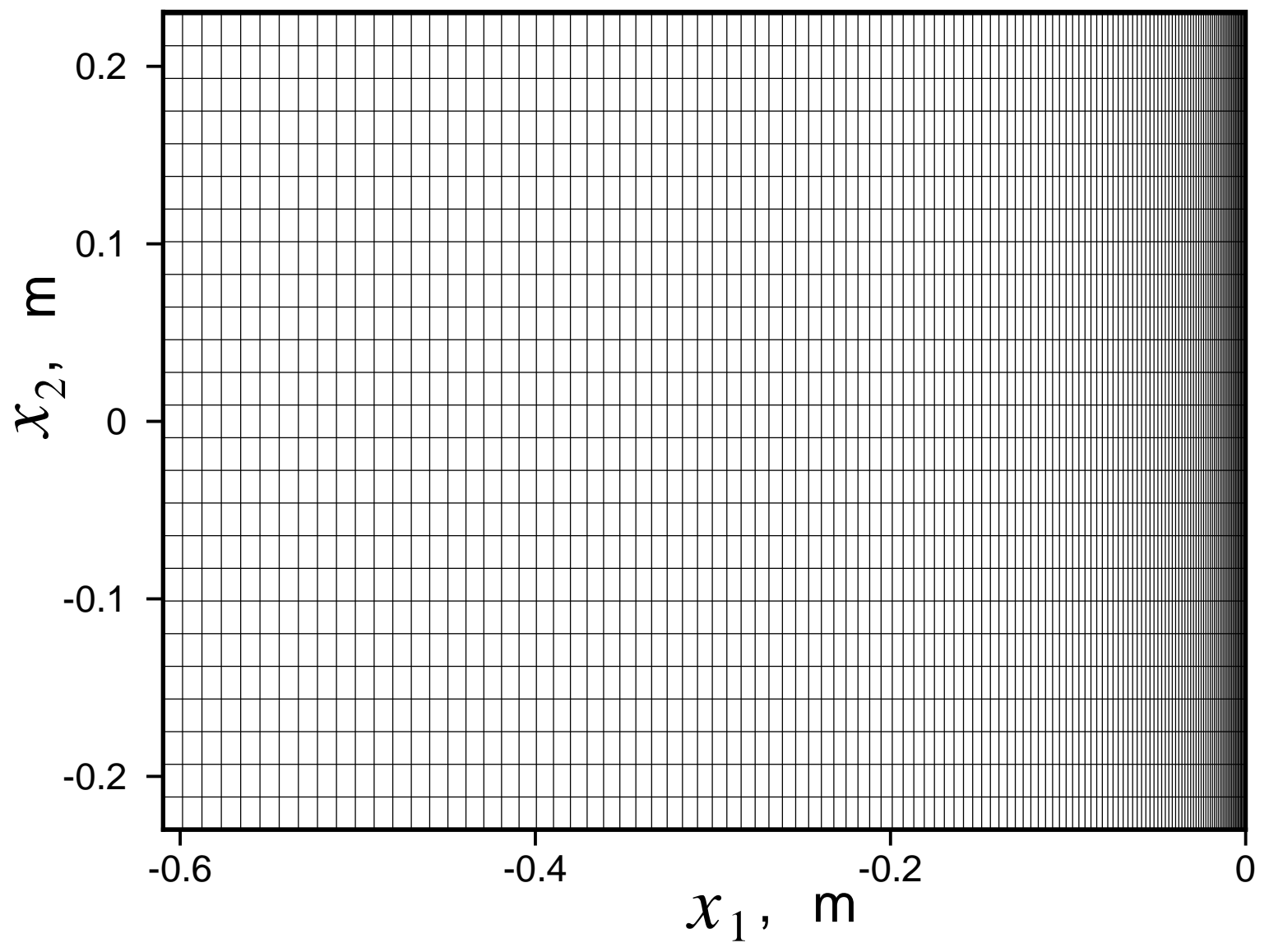

Figure 9 Surface grid for prediction of experiment of Brooks and Hodgson [17]; every fourth point of in each direction is shown. 


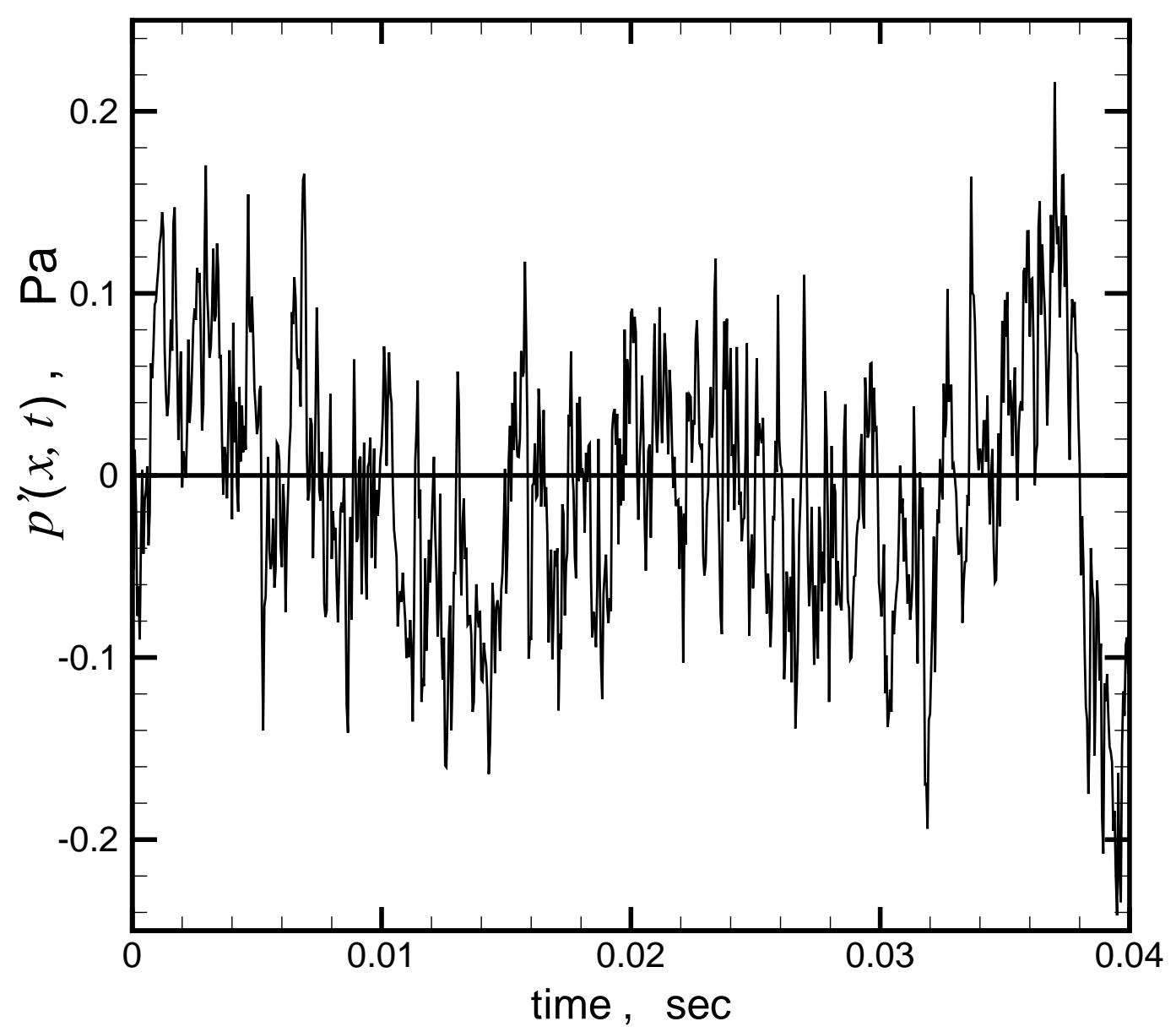

Figure 10 Predicted far field signal, $U=69.5 \mathrm{~m} / \mathrm{s} ;$ microphone at $90^{\circ}, 1.22 \mathrm{~m}$ above trailing edge. 


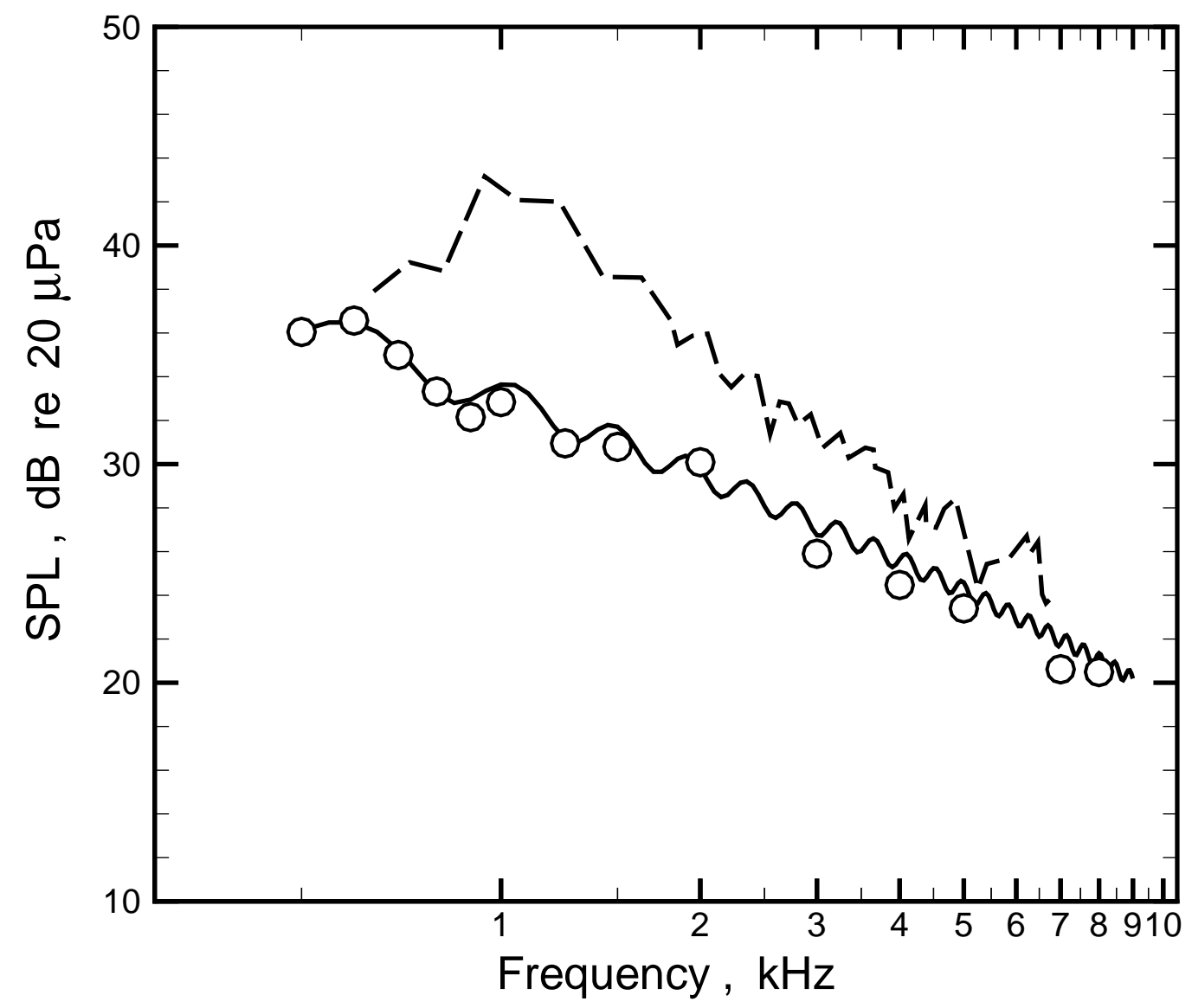

Figure 11 Predicted and measured far field noise spectra; predictions performed with surface pressure correlations from flat plate theory; $U=69.5 \mathrm{~m} / \mathrm{s} . \quad \bigcirc$, frequency domain prediction from $[16] ;-$, time domain domain prediction; --- , experimental measurement from [17]. 


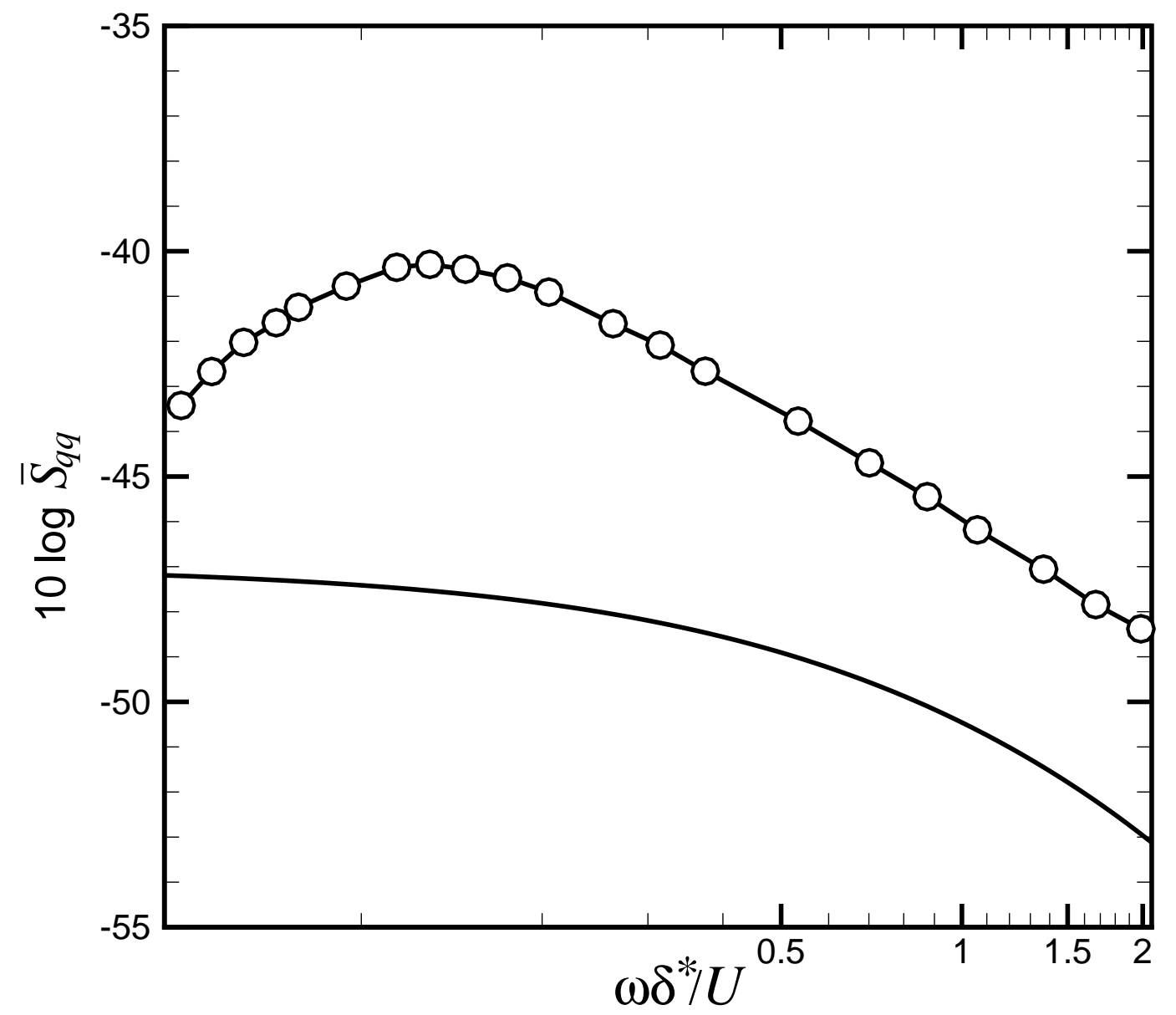

Figure 12 Normalized surface pressure correlations. — , from flat plate theory (Eq. (9a)); - , experimental measurements from [15]. 


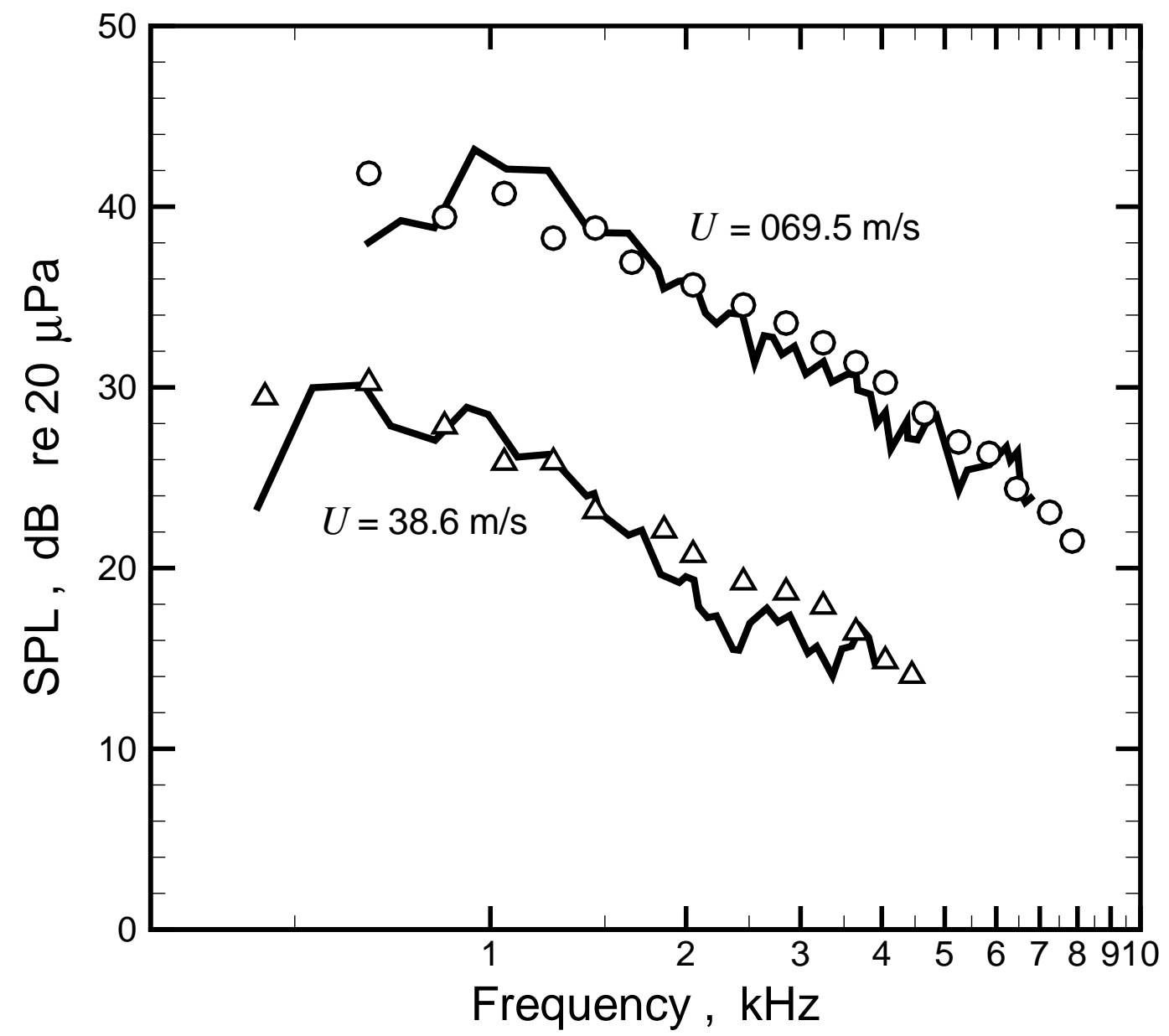

Figure 13 Predicted and measured far field noise spectra; predictions derived from time domain formulation (Eq. 6) and measured surface pressure correlations [15]. $\triangle$, prediction for $U=38.6$ $\mathrm{m} / \mathrm{s} ; \quad \bigcirc$, prediction for $U=69.5 \mathrm{~m} / \mathrm{s} ; \_$, experimental SPLs from [17]. 